\title{
Prototype Silicon Position-Sensitive Detector Working with Bragg-Brentano Powder Diffractometer
}

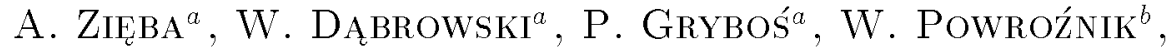 \\ T. Stobiecki ${ }^{b}$, K. ŚWIenteK ${ }^{a}$, J. SŁoWIK ${ }^{a}$ And P. WiąCEK ${ }^{a}$ \\ ${ }^{a}$ Faculty of Physics and Nuclear Techniques \\ University of Mining and Metallurgy \\ Al. Mickiewicza 30, 30-059 Kraków, Poland \\ ${ }^{b}$ Department of Electronics, University of Mining and Metallurgy
}

Al. Mickiewicza 30, 30-059 Kraków, Poland

\begin{abstract}
A prototype 64-channel detector module, comprising a silicon strip detector with strip pitch of $100 \mu \mathrm{m}$ and 64-channel ASIC RX64, was tested with the X-Pert Philips MPD diffractometer. Basic parameters of the detector module, energy resolution, and detection efficiency, were evaluated as a function of the counting rate. Energy resolution of $1.1 \mathrm{keV}$ FWHM for photon rate up to $1 \times 10^{7}$ photon $/ \mathrm{s}$ per $1 \mathrm{~cm}$ of the active width of the detector was demonstrated. The prototype detector, when applied in a diffractometer utilizing Bragg-Brentano focusing principle, allows to increase the counting rate by about 2 orders of magnitude with respect to a single counter. Exemplary diffraction patterns of polycrystalline samples of $\mathrm{Si}$ and $\mathrm{SiO}_{2}$ (quartz peak cluster) are presented.
\end{abstract}

PACS numbers: 07.85.-m, 29.40.Wk

\section{Introduction}

Silicon strip detectors are widely used for position-sensitive detection of charged particles in high energy particle physics experiments. Since a decade they are applied for position-sensitive detection of X-ray photons and represent a new class of one-dimensional position-sensitive single-photon counting devices. A strip detector is an array of $p-n$ reverse biased diodes shaped as narrow strips and made on common high-resistivity $n$-type silicon substrate. Such detectors require 
each strip to be read out by an individual electronic channel which is possible employing the technique of application specific integrated circuits (ASICs).

This paper describes application of a prototype 64-channel silicon strip detector to a Bragg-Brentano diffractometer, i.e., the most common type of X-ray diffraction apparatus. We demonstrate that in applications to such diffractometer, silicon strip detectors have several advantages not only when compared to the classical arrangement with a single counter but also with respect to other types of position-sensitive detectors.

\section{Silicon position-sensitive detector}

The prototype 64-channel detector module described in this work comprises two monolithic silicon structures: a strip detector with strip pitch of $100 \mu \mathrm{m}$ and a 64-channel readout ASIC RX64 [1]. The two chips are assembled on a small printed circuit board and connected together with wire bonding.

The 64-channel readout ASIC RX-64 [1] is the latest prototype being designed and fabricated in the frame of an ongoing program on development of read-out electronics for silicon strip detectors for X-ray detection. Main steps, compared to the previous 32-channel prototypes [2,3], is integration of the analogue front-end circuitry and the digital part, comprising counters and readout logic, in a single chip and extension up to 64 channels. This integration has improved significantly the robustness of the design and allowed for implementing some new features like internal calibration circuitry and internal digital-to-analogue converter (DAC) to control the threshold of the amplitude discriminators in the front-end circuits from an external computer. Thus, the detector module requires only a digital I/O PC card and the standard power supplies for standard data taking as well as for detailed measurements of performance.

The parameters of the detector module are summarised in Table. One parameter, namely the threshold uniformity deserves an additional comment. A key point in the architecture of the RX64 design is a common discrimination threshold applied to all channels. This solution works providing the channel-to-channel spread of the discriminator offsets is lower compared to the noise so that the effective threshold for each individual channel is not affected by the offset. Since the noise and the threshold spread are both random values and they add in square, the threshold spread (being a half of the noise) is practically negligible. The energy resolution given in Table takes into account both, the resolution of individual channel and the spread of channel parameters.

Basic parameters of the detector module, energy resolution and detection efficiency, was evaluated as a function of the counting rate. Energy resolution of $1.1 \mathrm{keV}$ FWHM for photon rate up to $1 \times 10^{7}$ photon/s per $1 \mathrm{~cm}$ of the active width of the detector was demonstrated. They were determined experimentally with the use of the radiation from X-ray tube (from $\theta-2 \theta$ diffractometer) and 
TABLE

Basic parameters of the detector module.

\begin{tabular}{l|c}
\hline \hline Number of channels & 64 \\
Strip pitch & $100 \mu \mathrm{m}$ \\
Length of the detector strips & $10 \mathrm{~mm}$ \\
Detector bias voltage & $100 \mathrm{~V}$ \\
Energy resolution & $1.1 \mathrm{keV} \mathrm{FWHM} \mathrm{(for} \mathrm{Cu} K_{\alpha}$ ) \\
Threshold uniformity & $0.5 \mathrm{keV} \mathrm{FWHM}$ \\
Maximum counting rate & $200 \mathrm{kHz} / \mathrm{channel}$ \\
Quantum efficiency & $99 \%$ for Cu $K_{\alpha}$ \\
& $\approx 50 \%$ for Mo $K_{\alpha}$ \\
Power consumption & $2.5 \mathrm{~mW} /$ channel \\
Cooling & not required
\end{tabular}

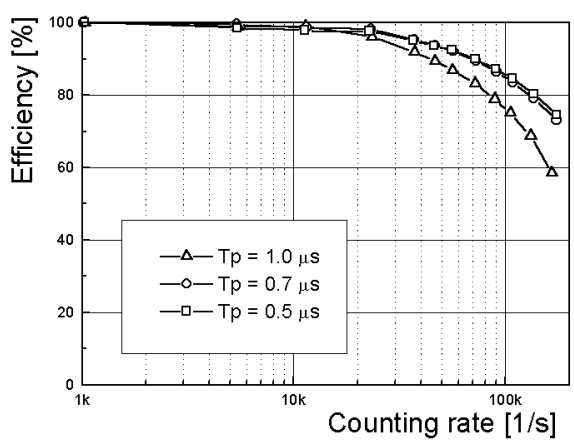

Fig. 1. Detector efficiency as a function of the counting rate.

suitable absorbers. In the RX-64 chip the shaping time $T_{\mathrm{p}}$ can be tuned in the range between $0.5 \mu \mathrm{s}$ and $1.0 \mu \mathrm{s}$. Using shorter shaping times the maximum counting rate can be increased in expense of somewhat higher noise. Figure 1 shows the measured efficiency as a function of the counting rate for three values of the shaping time. The maximum counting rate given in Table is tentatively defined as corresponding to the number of counts per second at which the efficiency decreases to $70 \%$ of the value at low intensity. The loss of efficiency at high counting rates is due to pile-up of pulses in the front-end circuitry and not due to charge collection time in the sensor, which is much shorter than the shaping times in the electronics. This counting rate limit concerns each channel separately and is not dependent on the total detector length, i.e., total number of readout channels. The above value corresponds to $20 \mathrm{MHz} / \mathrm{cm}$, a number which is 2 orders of magnitude higher than for gas position-sensitive detectors (PSDs). 


\section{Silicon strip detector in $\theta-2 \theta$ diffractometer}

Projects on application of silicon strip detectors for powder X-ray diffraction described in literature $[4,5]$ concerned Debye-Scherrer method. In this non-focusing geometry the diffracted photons are to be gathered in angular range close to $180^{\circ}$. Hence, the planned systems consist of thousand channels. We have developed the new application for the diffractometer based on Bragg-Brentano focusing principle $[6,3]$.

Application of gas PSDs to Bragg-Brentano powder diffractometer were described by Göbel [7] and Cheary and Coelho [8]. The gaseous PSDs (with a central wire electrode, typical length of $50 \mathrm{~mm}$ ) are optionally offered by manufacturers of the diffractometers [9]. The use of silicon strip PSD offers several advantages over the gas PSD, for example:

- better spatial resolution exclusively determined by the strip geometry. Our experiments $[2,3]$ have shown that the resolution of the diffraction pattern taken with the detector with strip pitch of $100 \mu \mathrm{m}$ is the same as for single-counter diffractometer with the receiving slit of $100 \mu \mathrm{m}$;

- better detection efficiency, specially evident for Mo $(17 \mathrm{keV})$ radiation;

- high counting rate per channel which leads to the values of counting rate per unit length which are two orders of magnitude higher than for the gas PSD.

The last advantage assures that the detector does not saturate in any diffraction experiment with conventional X-ray source. This makes possible to decrease the measurement's time of the typical polycrystalline samples from hours to minutes. The gas PSDs were practically used to speed up the prolonged measurements with the low intensity of diffracted beam (small samples, thin films).

The PSD is located on the detector arm of the diffractometer with the centre coinciding with the focusing circle. Registration of the diffraction beam at the finite distance $e$ from the focusing point gives rise to the distortion of the peak profile.

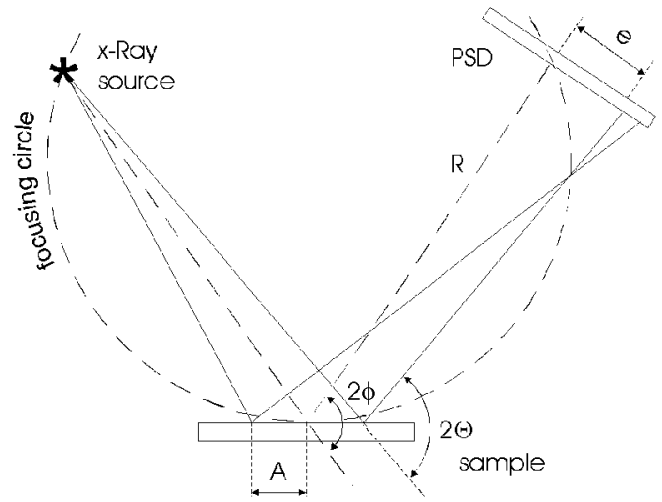

Fig. 2. Geometry of $\theta--2 \theta$ diffractometer working with the linear position-sensitive detector. 
(See Fig. 2; note that for $e \neq 0$ the instrument setting angle $2 \phi$ differs with respect to the diffraction angle 20.) The theory of geometrical equatorial aberration [10] makes it possible to predict both the peak profile and parameters describing that aberration. The contribution to a peak broadening is given (in term of rms peak width) by $\frac{1}{\sqrt{3}} \frac{A e}{R} \cos \phi$, where $R$ is the instrument radius and $2 A$ the illuminated length of the sample. Numerical calculation shows that for the detector already used the peak broadening remains negligible, but should be taken into account for future larger systems and small diffraction angles.

In present experiments the background level is comparable to that of traditional diffractometer without crystal monochromator. The background can be reduced by the combination of the beta filter, the use of antiscatter slits and the electronic two-level discrimination. The experiment described in Ref. [2] shows that the use of silicon strip detector with two-level discrimination allows to obtain the background level of the diffraction pattern slightly lower than that for the classical powder diffractometer with beta filter.

\section{Results}

The prototype detector was mounted on the detector arm of an X-Pert Phillips MPD diffractometer. With the instrument radius of $230 \mathrm{~mm}$ the detector covers angular range of $1.6^{\circ}$.

In order to obtain a diffraction pattern in large angular range one has to work with "step by step" mode scan. Figure 3 shows such a result for Si standard sample. With 40 steps the total measuring time was 10 min.

Observation of the structure change can be made with the fixed position of the PSD. As an example Fig. 4 shows the diffraction pattern for the so-called "quartz peak cluster". This feature results from the combination of 122,203 , and 301 Bragg peaks of $\mathrm{SiO}_{2}$ with $K_{\alpha_{1}}-K_{\alpha_{2}}$ splitting and is considered as a standard for comparison of spatial resolution. Measuring time was $3 \mathrm{~min}$.

\section{Outlook}

In the next step we plan to use two 64-channel RX-64 chips for readout of 128 strips simultaneously so the device will be $12.8 \mathrm{~mm}$ wide. We consider this size as minimal, which may practically compete in powder diffractometers with existing gas position-sensitive detectors. The $12.8 \mathrm{~mm}$ detector with a suitable data acquisition system will be a complete unit for diffractometry. Thanks to high efficiency it will be useful for measurements of thin films and multilayer structures where volume of samples is very small (grazing incidence diffraction (GID) and total reflectivity measurements).

Further development of the front-end electronics includes introducing two-level discrimination and increase in the counting rate. High counting rate capa- 


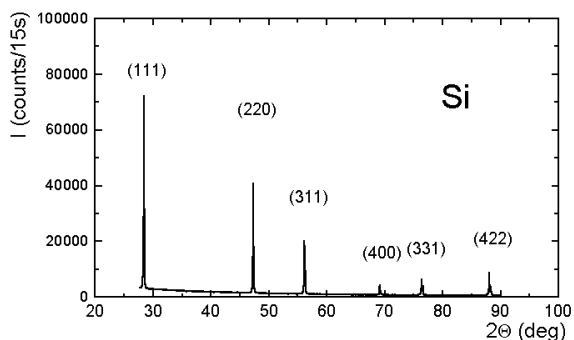

Fig. 3

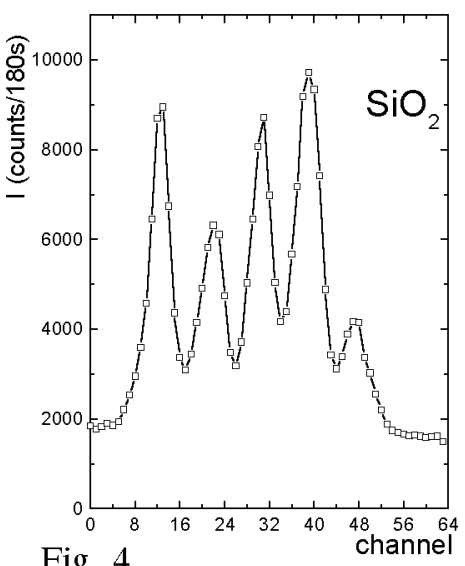

Fig. 4

Fig. 3. Diffraction pattern of Si standard polycrystalline sample.

Fig. 4. Diffraction pattern of the "quartz peak cluster". The scale from 1 to 64 channels corresponds to the interval of $2 \theta$ from $67.2^{\circ}$ to $68.8^{\circ}$.

bility, which can be increased by proper tuning of the front-end design, makes the device attractive for applications in experiments using synchrotron radiation.

\section{References}

[1] W. Dąbrowski, Nucl. Instrum. Methods Phys. Res. A 383, 179 (1996).

[2] P. Gryboś, W. Dą̧browski, IEEE Trans. Nucl. Science 48, 466 (2001).

[3] A. Zięba, W. Białas, W. Dąbrowski, P. Gryboś, M. Idzik, B. Leśniewska, J. Kudłaty, J. Słowik, in: Applied Crystallography. Proc. XVIII Conf., Eds. H. Morawiec, D. Stróż, World Scientific, Singapore 2000, p. 130.

[4] D. Loukas, V. Psycharis, E. Karvelas, A. Pavlidis, N. Haralabidis, J. Mousa, IEEE Trans. Nucl. Science 47, 877 (2000).

[5] F. Fauth, C. Bronnimann, H. Auderset, G. Maehlum, P. Pattison, B. Patterson, Nucl. Instrum. Methods Phys. Res. A 439, 138 (2000).

[6] A. Zięba, W. Dąbrowski, A. Czermak, in: The First Polish Meeting on High-Resolution X-Ray Diffraction and Topography, Szklarska Poręba (Poland) 1996, Conference Notes, High Pressure Research Centre, PAS, Warszawa 1996 (in Polish).

[7] H.E. Göbel, Adv. X-Ray Anal. 22, 255 (1979).

[8] R.W. Cheary, A.A. Coelho, J. Appl. Crystallogr. 27, 673 (1994).

[9] Position Sensitive Detector PSD-50M, Braun, Commercial Folder, 1999.

[10] J. Słowik, A. Zięba, J. Appl. Crystallogr. 34, 458 (2001). 